\title{
Determinants and underlying causes of frequent attendance in midwife-led care: an exploratory cross-sectional study
}

Janneke T. Gitsels - van der Wal ${ }^{1 *}$ (D), Lisanne A. Gitsels ${ }^{2}$, Angelo Hooker ${ }^{3,4}$, Britte van Weert ${ }^{1}$, Linda Martin ${ }^{1}$ and Esther I. Feijen - de Jong ${ }^{1,5}$

\begin{abstract}
Background: An adequate number of prenatal consultations is beneficial to the health of the mother and fetus. Guidelines recommend an average of 5-14 consultations. Daily practice, however, shows that some women attend the midwifery practice more frequently. This study examined factors associated with frequent attendance in midwifery-led care.

Methods: We conducted a cross-sectional study in a large midwifery practice in the Netherlands among low-risk women who started prenatal care in 2015 and 2016. Based on Andersen's behavioral model, we collected data on potential determinants from the digital midwifery's practice database. Prenatal healthcare utilization was measured by a revised version of the Kotelchuck Index, which measures a combination of care entry and numbers of visits. Logistic regression models were fitted to estimate the likelihood of frequent attendance compared to the recommended number of visits, adjusted for all relevant factors. Separate models were fitted on the non-referred and the referred group of obstetric-led care, as referral was found to be an effect modifier.

Results: The prevalence of frequent attendance was 23\% (243/1053), mainly caused by worries and/or vague complaints (44\%; 106/243). Among non-referred women, 53\% (560/1053), frequent attendance was associated with consultation with an obstetrician $(\mathrm{OR}=3.99(2.35-6.77))$ and exposure to sexual violence $(\mathrm{OR}=2.17(1.11-4.24))$. Among the referred participants, 47\% (493/1053), frequent attendance was associated with a consultation with an obstetrician $(\mathrm{OR}=2.75(1.66-4.57))$, psychosocial problems in the past or present $(\mathrm{OR}=1.85(1.02-3.35)$ or $\mathrm{OR}=2.99$ (1.43-6.25)), overweight $(\mathrm{OR}=1.88(1.09-3.24))$, and deprived area $(\mathrm{OR}=0.50(0.27-0.92))$.

Conclusion: Our exploratory study indicates that the determinants of frequent attendance in midwifery-led care differs between non-referred and referred women. Underlying causes for frequent attendance was mainly because of non-medical reasons. Implication for practice: A trustful midwife-client relationship is known to be needed for clients such as frequent attenders to share more detailed, personal stories in case of vague complaints or worries, which is necessary to identify their implicit needs.
\end{abstract}

Keywords: Midwifery, Prenatal care, Patient acceptance of health care, Social determinants of health, Sexual violence, Psychosocial deprivation

\footnotetext{
* Correspondence: jt.vanderwal@vumc.nl; Janneke@hllp.nl

${ }^{1}$ Amsterdam UMC, Vrije Universiteit Amsterdam, Midwifery Science, AVAG,

Amsterdam Public Health research institute, De Boelelaan 1117, Amsterdam

The Netherlands

Full list of author information is available at the end of the article
}

(c) The Author(s). 2019 Open Access This article is distributed under the terms of the Creative Commons Attribution 4.0 International License (http://creativecommons.org/licenses/by/4.0/), which permits unrestricted use, distribution, and reproduction in any medium, provided you give appropriate credit to the original author(s) and the source, provide a link to the Creative Commons license, and indicate if changes were made. The Creative Commons Public Domain Dedication waiver (http://creativecommons.org/publicdomain/zero/1.0/) applies to the data made available in this article, unless otherwise stated. 


\section{Background}

In 2016 the World Health Organisation advised at least eight prenatal visits during pregnancy to decrease perinatal mortality and to increase positive experiences of pregnant women [1]. The National Institute for Health and Care Excellence (NICE) advises three additional visits for nulliparous women [2]. Despite these recommendations, the number of face-to-face visits varies between five and fourteen worldwide [1-4]. The current prenatal care schedule of low-risk pregnant women in the Netherlands comprises an average of 14 visits in pregnancy, the highest number of visits for low-risk women worldwide [5]. In addition, the Royal Dutch Organisation of Midwives (KNOV - Koninklijke Nederlandse Organisatie van Verloskundigen) recommends adjusting the number of prenatal visits to the medical care, needs, and preferences of the woman. More visits could be initiated by the care provider as well as by the caretaker [5-7].

Frequent attendance, defined as patients receiving significantly more visits than recommended, has hardly been studied in midwifery-led care whereas it is a recognized problem in general practice and hospitals. For instance, $10 \%$ of patients account for approximately $40 \%$ of encounters to general practices $[8,9]$. Frequent attendance has been associated with psychosocial problems such as anxiety, high perceived stress, low self-esteem and poor self-regulation $[8,9]$. Women are more often frequent attenders than men [10]. Feijen-de Jong et al. investigated prenatal health care utilization in primary midwifery care in a multicentre prospective dynamic cohort study in the Netherlands and showed that $66 \%$ of the participants had received the recommended number of visits, $21 \%$ fewer, and $13 \%$ more [3]. A Swedish study showed that $25 \%$ received the recommended number of visits, $17 \%$ fewer and $57 \%$ more [7].

Previous studies have found a positive association between frequent attendance and preterm birth, low birth-weight, perinatal mortality, lower Apgar scores, induction of labour and caesarean section [11-14]. Due to the increasing number of women seem to be having more visits than recommended, resulting in increased workload of midwives and their clinics without apparent improvement of outcomes, it is essential to understand why some women require more visits than others; understanding this phenomenon can contribute to the improvement of the healthcare organization, planning and execution of care and most importantly, might improve perinatal outcomes. The aim of this study was therefore to examine the prevalence, determinants, and underlying causes of frequent attendance in midwife-led care, and whether these differ from those who are referred to an obstetrician. We used Andersen's behavioral model of healthcare use as a guiding framework to categorize the determinants of health care utilization. This model suggests that the use of health care depends on predisposing, enabling, need and health behavior factors [15].

\section{Dutch healthcare system}

The care for pregnant women in the Netherlands is organized as follows: low-risk pregnant women receive prenatal, natal and postnatal care from a midwife, high-risk women receive care from an obstetrician while women with intermediate risk received joint care. In case of a change in the medical condition of a low-risk woman, an obstetrician is consulted and in case of a determined medical condition, the woman is referred to an obstetrician, who will provide care during the remaining pregnancy period and birth. Otherwise, the woman is referred back to the midwife.

Women with psychological problems are often referred to the Psychiatrist-Obstetrician-Pediatrician-clinic, an outpatient setting where pregnant women are evaluated by obstetricians, pediatricians, and psychologists and receive advice from this multi-disciplinary team. For the clarity of this paper, the word 'visit' is used related to midwifery-led care, the word 'consultation' is used related to consultation of an obstetricians and other specialists in hospitals.

\section{Methods \\ Study design}

This exploratory cross-sectional study was conducted in a large midwifery practice, located in a medium-sized city near Amsterdam, the Netherlands. The practice was organized in two teams, each consisting of four midwives. On the 25th of January 2018, the Medical Ethics Committee of the VU University Medical Center (ref. 2018.019) approved this study to collect and analyse retrospective data of January 2015 to January 2017. Women gave permission to use their anonymous data, their verbal consent was obtained during the intake and noted in their files while they watched; the beforementioned Medical Ethics Committee approved this procedure of obtaining consent.

\section{Participants and measurement tool}

Eligible participants were pregnant women at the specified midwifery practice during the study period with an ongoing pregnancy. Women with a miscarriage and women who only received postnatal care were excluded. Included participants were classified based on the level of health care utilization according to the Kotelchuck Index-Revised, see Appendix [3, 12].

In brief, the Kotelchuck Index-Revised, a validated tool for measuring the level of care utilization, is based on the number of face-to-face visits with the midwife, adjusted for gestation and onset of care and aligned with the number of recommended face-to-face prenatal visits by the KNOV. Utilization of care was defined as inadequate (less 
than $50 \%$ of the recommended visits), intermediate (50$79 \%)$, adequate $(80-109 \%)$ and adequate plus $(110 \%$ or more) $[3,12]$. In this study, women with adequate plus utilization of care were considered to be frequent attendance (cases) and were compared to women with the adequate utilization of care (controls). Women with the intermediate or inadequate utilization of prenatal care were excluded from the study because these groups were irrelevant in view of the goal of our study.

\section{Data collection}

The digital midwifery database was searched to identify eligible participants. Two researchers (JG and BW) extracted data from the database, which were anonymized and de-identified. In this database, the medical charts of all pregnant women guided by this midwifery practice are included. The chart includes medical care records of pregnancies, demographic characteristics, lifestyle and medical information of the pregnant woman. Possible reasons for frequent attendance were identified based on the additional information in the charts of frequent attenders documented by the midwife.

Based on the Andersen's model, several variables concerning predisposing, enabling, need and health behavior factors were considered as potential determinants of frequent attendance $[3,15]$. In this study, Predisposing variables consisted of sociodemographic factors such as age, ethnicity (Dutch, western non-Dutch, non-western non-Dutch), marital status (relationship yes/no), occupation (yes/no), and level of education. The level of education was subdivided into low (primary school and some vocational training), intermediate (secondary school and completed vocational training), and high (college and/or university). Enabling variables included deprivation (yes/ no), year of the start of prenatal care (2015/2016), and team (team 1 or team 2); both teams consisted of four midwives. Needs variables consisted of perceived and evaluated needs. Perceived needs in this study were telephone consultations initiated by clients (numbers), psychosocial problems (no/yes in the past, yes at present), and sexual violence (no/yes). Evaluated needs included parity (nulliparous or multiparous), the start of pregnancy (spontaneous or assisted), duration of getting pregnant (in months), reasons for frequent attendance, attendance to health care professionals other than the midwife, the number of ultrasound examinations, and prescribed drugs. Reasons for frequent attendance were defined based on the information in clients' dossiers. Consultation of other healthcare professionals was subdivided in visits with a general practitioner, a medical specialist e.g. an obstetrician or an internist, or a visit to the Psychiatrist-Obstetrician-Pediatrician-clinic (POP). Health behavior variables consisted of smoking (no/yes), alcohol consumption (no/yes), intake of folic acid (no/ yes), and body mass index before pregnancy (BMI). We classified BMI according to the World Health Organization Classification of adults: underweight and normal weight, overweight, and obesity [16].

\section{Analyses}

Descriptive analysis included contingency tables to summarize the study population. Categorical data were reported as absolute numbers and percentages. Normally distributed continuous variables were reported as means with standard deviations and non-normally distributed continuous variables as medians with interquartile ranges.

Logistic regression models were fitted to estimate the unadjusted and adjusted effects of frequent attendance associated with the variables described above. Variables with a prevalence of less than $5 \%$ were excluded from the regression analysis. The team was included in the models as a fixed effect because the two teams provided care to an equal number of participants. Age was re-coded to be centered around the mean to have a meaningful baseline. Backward elimination was performed to obtain the leanest model possible, where each time, the variable with the largest $p$-value was removed from the model until all variables were significant $(p<.05)$. The model assumptions were checked and the final model was assessed on overall performance (Nagelkerke's R2) and discrimination (specificity, sensitivity, and overall accuracy).

Referral to obstetrician was considered an effect modifier of prenatal healthcare utilization. 3 Therefore, additional analyses were performed comparing referred and non-referred pregnant women with $\chi^{2}$ tests for categorical variables and separate logistic models were fitted on the subsets of non-referred and referred participants. All analyses were executed using SPSS (version 24).

\section{Results}

Between January 2015 and January 2017, 1497 women received prenatal and/or postnatal care by the midwifery practice. The flow chart of the eligible study population is shown in Fig. 1. The final study population consisted of 1053 women: 810 (77\%) received adequate healthcare, and $243(23 \%)$ received adequate plus health care (frequent attendance).

\section{Results of the complete study group}

The characteristics of the study population are shown in Table 1, column 2. The prevalence of frequent attendance in 2015 and 2016 were 19 and 24\%, respectively. In our cohort, the average age was 29.2 years, $48 \%$ of the women were primipara was 48 , and $53 \%$ had a non-Dutch background. Of all women, 96 (9\%) participants reported to use prescribed drugs, mainly Selective Serotonin Reuptake Inhibitors and thyroid medication. 


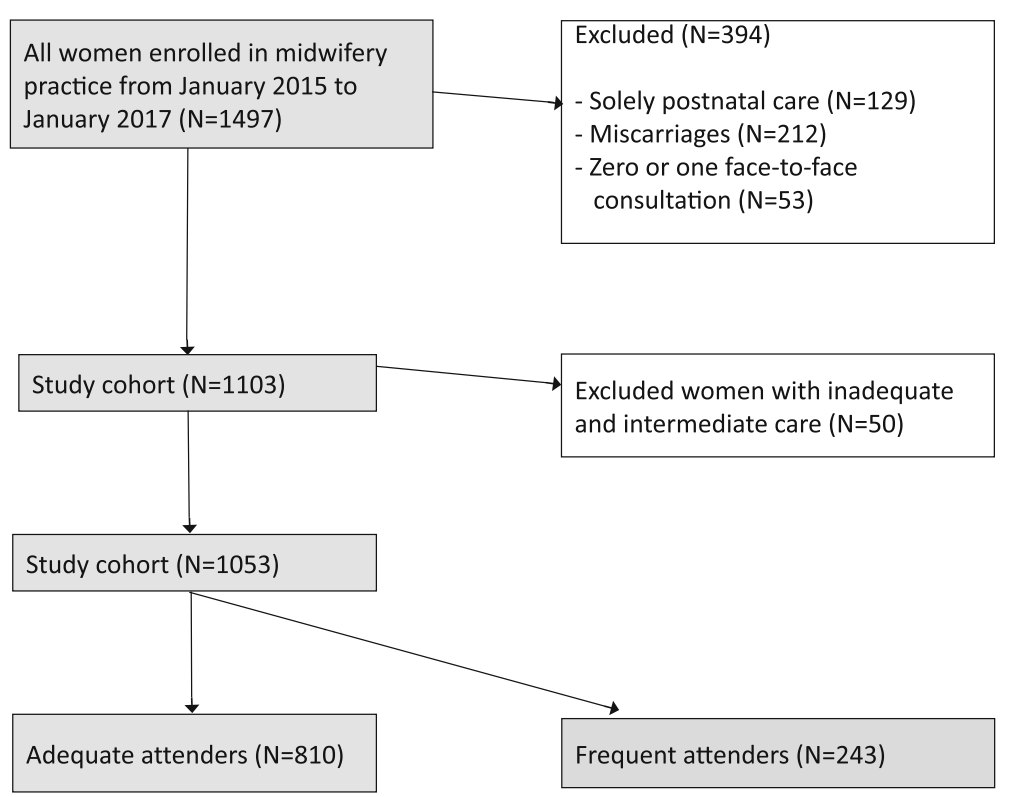

Fig. 1 Eligible study population. The flow chart of the eligible study population

The underlying reasons for more than the recommended visits in the midwifery practice in the 243 frequent attenders, independent of referral, are shown in Table 2. The most reported reasons were being worried/having vague complaints (44\%), followed by abdominal pain (15\%), medical problems (13\%), psychosocial problems, e.g. behavioral, emotional and/or relational problems $(12 \%)$ and experiencing high pregnancy burden (8\%).

Table 3 shows reasons for consultation with an obstetrician and reasons for referral to an obstetrician. More than half of the study population, 572/1053 (55\%), had at least one consultation with an obstetrician, ranging from one to seven; the mean of the number of consultations for frequent attenders and adequate users was 2.7 (SD 2.97) and 1.9 (SD 4.83), respectively. Of the whole study population, 493/ $1053(47 \%)$ were referred to an obstetrician. Half of the women referred, 252/493 (51\%) had at least one prior consultation with an obstetrician. Frequent attenders were more likely to be referred compared to adequate users (respectively 54.7 and 44.4\%; $p=$ 0.005). Women of non-western non-Dutch origin were more likely to be referred compared to women of Dutch or women of western non-Dutch origin (respectively 51.6, 44.8, and 31.8\%; $p=0.002$ ). Women living in a deprived area were more likely to be referred, compared to women not living in a deprived area (respectively 55.8 and 44.6\%; $p=0.003$ ).

The final logistic regression model on the entire study population included enabling, need, and health behaviors variables, but no predisposing variables. Women who started their prenatal care in 2016, with an assisted start of pregnancy, a consultation with an obstetrician, a history of sexual violence, more than average ultrasounds, and were overweight had higher odds of frequent attendance, whereas women living in a deprived area had lower odds of frequent attendance (Table 4).

Analyses showed referral to an obstetrician as an effect modifier, therefore, we split the results by referral (Table 4).

\section{Results of non-referred pregnant women}

The characteristics of the non-referred population (frequent attenders and adequate users) are shown in Table 1 , columns 3 and 4 .

The final logistic regression model on the subset of non-referred participants included only need variables: consultation with an obstetrician and a history of sexual violence. Women with one or more consultations with an obstetrician and women with a history of sexual violence had higher odds of frequent attendance (Table 4, columns 4 and 5).

\section{Results of referred pregnant women}

The characteristics of the referred population (frequent attenders and adequate users) are shown in Table 1, columns 5 and 6 .

The final logistic regression model on the subset of referred participants included enabling, need, and health behavior variables: deprived area, year, 
Table 1 Various characteristics of women with adequate plus or adequate use of prenatal care, split by referrals to an obstetrician in the study practice $(N=1053)$

\begin{tabular}{|c|c|c|c|c|c|}
\hline & Study population $N=1053(\%)$ & $\begin{array}{l}\text { Non-referred } \\
\text { Adequate plus } \\
N=110(10.4 \%)\end{array}$ & $\begin{array}{l}\text { Non-referred } \\
\text { Adequate } \\
N=450(42.7 \%)\end{array}$ & $\begin{array}{l}\text { Referred } \\
\text { Adequate plus } \\
N=133(12.6 \%)\end{array}$ & $\begin{array}{l}\text { Referred } \\
\text { Adequate } \\
N=360(34.2 \%)\end{array}$ \\
\hline \multicolumn{6}{|l|}{ Healthcare utilization } \\
\hline Adequate plus (cases) & $243(23.1)$ & & & & \\
\hline Adequate (controls) & $810(76.9)$ & & & & \\
\hline \multicolumn{6}{|l|}{ Referrals } \\
\hline Non-referred & $560(53.2)$ & & & & \\
\hline Referred & $493(46.8)$ & & & & \\
\hline \multicolumn{6}{|l|}{ Consultations face-to-face } \\
\hline Mean (sd) & $10.8(4.2)$ & $15.5(3.6)$ & $11.8(2.7)$ & $12.5(4.2)$ & $7.7(3.6)$ \\
\hline \multicolumn{6}{|l|}{ Predisposing variables } \\
\hline \multicolumn{6}{|l|}{ Age in years } \\
\hline Mean (sd) & $29.2(4.2)$ & $28.2(5.0)$ & $28.7(4.7)$ & $29.3(5.1)$ & $30.1(4.9)$ \\
\hline \multicolumn{6}{|l|}{ Level of Education ${ }^{\mathrm{a}}$} \\
\hline Low & $121(11.9)$ & $8(7.5)$ & $49(11.2)$ & $18(14.3)$ & $46(13.1)$ \\
\hline Middle (intermediate) & $479(47.0)$ & $55(51.4)$ & $203(46.6)$ & $58(46.0)$ & $163(46.6)$ \\
\hline High & $419(41.1)$ & $44(41.1)$ & $184(42.2)$ & $50(39.7)$ & $141(40.3)$ \\
\hline \multicolumn{6}{|l|}{ Ethnic Background ${ }^{a}$} \\
\hline Dutch & $500(47.5)$ & $55(50.0)$ & $221(49.1)$ & $74(55.6)$ & $150(41.8)$ \\
\hline Western Non-Dutch & $85(8.1)$ & $9(8.3)$ & $49(10.9)$ & $5(3.8)$ & $22(6.1)$ \\
\hline Non-Western Non-Dutch & $467(44.4)$ & $46(41.8)$ & $180(40.0)$ & $54(40.6)$ & $187(52.1)$ \\
\hline \multicolumn{6}{|l|}{ Marital status } \\
\hline Single & $27(2.6)$ & $4(3.6)$ & $14(3.1)$ & $5(3.8)$ & $4(1.1)$ \\
\hline Married/partner & $1026(97.4)$ & $106(96.4)$ & $436(96.9)$ & $128(96.2)$ & $356(98.9)$ \\
\hline \multicolumn{6}{|l|}{ Occupation $^{a}$} \\
\hline Unemployed & $326(31.2)$ & $33(30.3)$ & $142(31.8)$ & $41(31.1)$ & $110(30.9)$ \\
\hline Employed & $718(68.8)$ & $76(69.7)$ & $305(68.2)$ & $91(68.9)$ & $246(69.1)$ \\
\hline \multicolumn{6}{|l|}{ Enabling variables } \\
\hline \multicolumn{6}{|l|}{ Deprived area ${ }^{a}$} \\
\hline No & $835(79.4)$ & $91(82.7)$ & $372(82.9)$ & $110(82.7)$ & $262(72.8)$ \\
\hline Yes & $217(20.6)$ & $19(17.3)$ & $77(17.1)$ & $23(17.3)$ & $98(27.2)$ \\
\hline \multicolumn{6}{|l|}{ Team } \\
\hline 1 & $530(50.3)$ & $64(58.2)$ & $224(49.8)$ & $71(53.4)$ & $171(47.5)$ \\
\hline 2 & $523(49.7)$ & $46(41.8)$ & $226(50.2)$ & $62(46.6)$ & $189(52.5)$ \\
\hline \multicolumn{6}{|l|}{ Year } \\
\hline 2015 & $510(48.4)$ & $53(48.2)$ & $224(49.8)$ & $51(38.3)$ & $182(50.6)$ \\
\hline 2016 & $543(51.6)$ & $57(51.8)$ & $226(50.2)$ & $82(61.7)$ & $178(49.4)$ \\
\hline \multicolumn{6}{|l|}{ Need variables } \\
\hline \multicolumn{6}{|l|}{ Consultations telephone } \\
\hline Mean (sd) & $2.8(3.2)$ & $4.7(4.5)$ & $2.1(2.4)$ & $4.8(4.3)$ & $2.3(2.4)$ \\
\hline \multicolumn{6}{|l|}{ Parity } \\
\hline Nulliparous & $509(48.3)$ & $59(53.6)$ & $222(49.3)$ & $66(49.6)$ & $162(45.0)$ \\
\hline Multiparous & $544(51.7)$ & $51(46.4)$ & $228(50.7)$ & $67(50.4)$ & $198(55.0)$ \\
\hline \multicolumn{6}{|l|}{ Start of pregnancy ${ }^{\mathrm{a}}$} \\
\hline Spontaneous & $989(94.9)$ & $104(96.3)$ & $429(96.4)$ & $117(88.6)$ & $339(95.0)$ \\
\hline Assisted & $53(5.1)$ & $4(3.7)$ & $16(3.6)$ & $15(11.4)$ & $18(5.0)$ \\
\hline
\end{tabular}

Duration of getting pregnant in months ${ }^{\mathrm{a}}$ 
Table 1 Various characteristics of women with adequate plus or adequate use of prenatal care, split by referrals to an obstetrician in the study practice $(N=1053)$ (Continued)

\begin{tabular}{|c|c|c|c|c|c|}
\hline & Study population N = 1053 (\%) & $\begin{array}{l}\text { Non-referred } \\
\text { Adequate plus } \\
N=110(10.4 \%)\end{array}$ & $\begin{array}{l}\text { Non-referred } \\
\text { Adequate } \\
N=450 \text { (42.7\%) }\end{array}$ & $\begin{array}{l}\text { Referred } \\
\text { Adequate plus } \\
N=133(12.6 \%)\end{array}$ & $\begin{array}{l}\text { Referred } \\
\text { Adequate } \\
N=360 \text { (34.2\%) }\end{array}$ \\
\hline mean (sd) & $5.2(10.2)$ & $5.2(10.1)$ & $4.1(7.9)$ & $6.7(13.0)$ & $5.9(11.2)$ \\
\hline \multicolumn{6}{|c|}{ Attendance general practitioner } \\
\hline No & $911(86.5)$ & $96(87.3)$ & $400(88.9)$ & $106(79.7)$ & $309(85.8)$ \\
\hline Yes & $142(13.5)$ & $14(12.7)$ & $50(11.1)$ & $27(20.3)$ & $51(14.2)$ \\
\hline \multicolumn{6}{|c|}{ Attendance Specialist } \\
\hline No & $757(71.9)$ & $82(74.5)$ & $342(76.0)$ & $81(60.9)$ & $252(70.0)$ \\
\hline Yes & $296(28.1)$ & $28(25.5)$ & $108(24.0)$ & $52(39.1)$ & $108(30.0)$ \\
\hline \multicolumn{6}{|c|}{ Consultation Obstetrician $^{\mathrm{a}}$} \\
\hline No & $478(45.5)$ & $22(20.0)$ & $217(48.3)$ & $36(27.1)$ & $203(56.7)$ \\
\hline Yes & $572(54.5)$ & $88(80.0)$ & $232(51.7)$ & $97(72.9)$ & $155(43.3)$ \\
\hline \multicolumn{6}{|c|}{ Psychosocial problems ${ }^{\mathrm{a}}$} \\
\hline No & $749(71.3)$ & $77(70.0)$ & $333(74.5)$ & $72(54.1)$ & $267(74.2)$ \\
\hline Yes in the past & $211(20.1)$ & $25(22.7)$ & $87(19.5)$ & $33(24.8)$ & $66(18.3)$ \\
\hline Yes at present & $90(8.6)$ & $8(7.3)$ & $27(6.0)$ & $28(21.1)$ & $27(7.5)$ \\
\hline \multicolumn{6}{|l|}{ POP consultation } \\
\hline No & $1018(96.7)$ & $104(94.5)$ & $443(98.4)$ & $121(91.0)$ & $350(97.2)$ \\
\hline Yes & $35(3.3)$ & $6(5.5)$ & $7(1.6)$ & $12(9.0)$ & $10(2.8)$ \\
\hline \multicolumn{6}{|c|}{ Sexual or domestic violence ${ }^{a}$} \\
\hline No & $915(87.1)$ & $91(82.7)$ & 407 (90.6) & $100(75.2)$ & $317(88.1)$ \\
\hline Yes & $136(12.9)$ & $18(16.5)$ & $42(9.4)$ & $33(24.8)$ & $43(11.9)$ \\
\hline \multicolumn{6}{|l|}{ Prescribed Drugs ${ }^{a}$} \\
\hline No & $955(90.9)$ & $101(91.8)$ & $420(93.5)$ & $114(85.7)$ & $320(89.1)$ \\
\hline Yes & $96(9.1)$ & $9(8.2)$ & $29(6.5)$ & $19(14.3)$ & $39(10.9)$ \\
\hline \multicolumn{6}{|c|}{ Number of ultrasounds } \\
\hline Mean (sd) & $3.9(1.4)$ & $4.1(1.3)$ & $3.9(1.4)$ & $4.2(1.5)$ & $3.7(1.5)$ \\
\hline \multicolumn{6}{|c|}{ Health behaviours variables } \\
\hline \multicolumn{6}{|l|}{ Smoking ${ }^{a}$} \\
\hline No & $806(76.7)$ & $77(70.0)$ & $363(80.8)$ & $88(66.2)$ & $278(77.4)$ \\
\hline Yes & $245(23.3)$ & $33(30.0)$ & $86(19.2)$ & $45(33.8)$ & $81(22.6)$ \\
\hline \multicolumn{6}{|l|}{ Alcohol use $\mathrm{a}^{\mathrm{a}}$} \\
\hline No & $1006(95.7)$ & $101(91.8)$ & $434(96.7)$ & $122(91.7)$ & $349(97.2)$ \\
\hline Yes & $45(4.3)$ & $9(8.2)$ & $15(3.3)$ & $11(8.3)$ & $10(2.8)$ \\
\hline \multicolumn{6}{|l|}{ Drugs addiction ${ }^{\mathrm{a}}$} \\
\hline No & $1028(97.8)$ & $109(99.1)$ & $441(98.2)$ & $124(93.2)$ & $354(98.6)$ \\
\hline Yes & $23(2.2)$ & $1(0.9)$ & $8(1.8)$ & $9(6.8)$ & $5(1.4)$ \\
\hline \multicolumn{6}{|l|}{$\mathrm{BMl} \mathrm{I}^{\mathrm{a}}$} \\
\hline Normal weight & $656(62.5)$ & $80(72.7)$ & $315(70.5)$ & $64(48.1)$ & $197(54.9)$ \\
\hline Overweight & $256(24.4)$ & $24(21.8)$ & $93(20.8)$ & $46(34.6)$ & $93(25.9)$ \\
\hline Obese & $137(13.1)$ & $6(5.5)$ & $39(8.7)$ & $23(17.3)$ & $69(19.2)$ \\
\hline
\end{tabular}

asample size varies due to missing data; valid percentages are shown; POP Psychiatrist-Obstetrician Pediatricianclinic; education: low (primary school and some vocational training), intermediate (secondary school and completed vocational training), and high (college and/or university)

consultation with an obstetrician, psychosocial problems, number of ultrasounds, and BMI (Table 4, columns 6 and 7). Women living in a deprived area had lower odds of frequent attendance, whereas women who started prenatal care in 2016, with one or more consultations with an obstetrician, 
Table 2 Underlying reasons for frequent attendance $(N=243)$

\begin{tabular}{ll}
\hline Reason & $N(\%)$ \\
\hline Worried/vague complaints & $106(43.6)$ \\
Abdominal complaints without medical explanation & $37(15.2)$ \\
Highchosocial problems & $29(11.9)$ \\
Hypertension complaints & $20(8.2)$ \\
Previous miscarriage & $11(4.5)$ \\
Long-term children wish & $11(4.5)$ \\
Blood loss first trimester & $8(3.3)$ \\
Blood loss second trimester & $7(2.9)$ \\
Nausea & $4(1.6)$ \\
Growth retardation & $4(1.6)$ \\
Hemoglobin pathology & $1(0.4)$ \\
A fetus with a congenital anomaly & $1(0.4)$ \\
Combination of reasons & $1(0.4)$ \\
Total & $3(1.2)$
\end{tabular}

psychosocial problems in the past or present, who had more than average ultrasounds, and were overweight had higher odds of frequent attendance.

\section{Discussion}

We assessed the prevalence, determinants and underlying causes of frequent attenders of prenatal care by referred and non-referred women in a large Dutch midwifery practice. This exploratory study showed that almost one in four pregnant women received adequate plus care and were considered frequent attenders. The strongest associations of frequent attendance in both the non-referred and the referred participants were consultation with an obstetrician. In the non-referred group a history of sexual violence, and in the referred group psychosocial problems showed the strongest associations. The underlying causes of frequent attendance were mainly due to worries and/or vague complaints and abdominal pain.

In our study, $23 \%$ of the study population meet the criteria and were considered frequent attendees and accounted for a significant number of prenatal visits. Our findings are in line with a large retrospective study in the United States that reported a frequent attendance rate of $30 \%$ among low-risk pregnant women [14]. Contrary, a Dutch national study reported a prevalence of frequent attendance of 13\% between 2009 and 2011 in primary midwifery care [3]. The higher frequent attendance rate in the current study could be explained by several interventions that were introduced since 2011 to
Table 3 Reasons for referral and consultation obstetrician among the entire study population $(N=1053)$

\begin{tabular}{|c|c|c|}
\hline Reasons & $\begin{array}{l}\text { Referral } \\
N= \\
493 \\
(46.8 \%)\end{array}$ & $\begin{array}{l}\text { Consultation } \\
N=574 \\
(54.5 \%)\end{array}$ \\
\hline Glucose intolerance & $\begin{array}{l}138 \\
(27.9)\end{array}$ & \\
\hline Pregnancy-induced hypertension & $\begin{array}{l}70 \\
(14.1)\end{array}$ & $48(8.4)$ \\
\hline Previous C-section & $48(9.7)$ & $22(3.8)$ \\
\hline Postterme ( $\geq 42$ weeks)/request for induction & $30(6.1)$ & $35(6.1)$ \\
\hline Breech or other malpresentation & $27(5.5)$ & $17(3.0)$ \\
\hline Less fetal movements & $23(4.6)$ & $81(14.1)$ \\
\hline Intercurrent disease & $18(3.6)$ & \\
\hline Small for gestational age & $17(3.4)$ & \\
\hline Premature birth & $15(3)$ & \\
\hline Suspicion of premature birth & & $20(3.5)$ \\
\hline Congenital anomaly & $12(2.4)$ & $13(2.3)$ \\
\hline Large for gestational age & $11(2.2)$ & \\
\hline Anaemia & $11(2.2)$ & $36(6.3)$ \\
\hline Previous small for gestational age & $9(1.8)$ & \\
\hline Placenta problems & $6(1.2)$ & \\
\hline Hydramnion & $6(1.2)$ & \\
\hline Uterus anomaly/cyst/myomas & $5(1)$ & \\
\hline Stillbirth & $4(.8)$ & \\
\hline Hyperemesis & $3(.6)$ & \\
\hline Fetal distress & $2(.4)$ & $16(2.8)$ \\
\hline Blood loss & $2(.4)$ & $39(6.8)$ \\
\hline Psychiatric diseases & $1(.2)$ & \\
\hline Psychiatric problems (POP) & & $12(2.1)$ \\
\hline Twin pregnancy & $1(.2)$ & \\
\hline Abdominal pains & & $74(12.9)$ \\
\hline Fetal growth control & & $68(11.8)$ \\
\hline
\end{tabular}

Other, such as cholestasis, maternal heart 34 (6.9)

defects, rheumatism, gastric bypass, pelvic pain

Other, such as pelvic pain, GBS, clotting disorder, vague complaints

$93(19.9)$

improve perinatal care. These interventions were introduced because of a relatively high perinatal mortality rate in the Netherlands compared to other European countries and were aimed at increasing awareness for psychosocial problems, e.g. implementation of the Psychiatrist-Obstetrician-Pediatrician -consultations, and mainly targeted at women living in deprived areas and women with a non-western non-Dutch background [18-20]. 
Table 4 Unadjusted and adjusted associations of various characteristics of women with adequate plus use of care, and split by subsequent referral in the study practice

\begin{tabular}{|c|c|c|c|c|c|c|}
\hline & \multicolumn{2}{|l|}{ Study population } & \multicolumn{2}{|l|}{ Not referred } & \multicolumn{2}{|l|}{ Referred } \\
\hline & $\begin{array}{l}\text { Unadjusted OR (Cl) } \\
n \leq 1053^{\mathbf{a}}\end{array}$ & $\begin{array}{l}\text { Adjusted OR (Cl) } \\
n=937\end{array}$ & $\begin{array}{l}\text { Unadjusted OR (Cl) } \\
n \leq 560^{\mathrm{a}}\end{array}$ & $\begin{array}{l}\text { Adjusted OR (Cl) } \\
n=502\end{array}$ & $\begin{array}{l}\text { Unadjusted OR (Cl) } \\
n \leq 493^{\mathrm{a}}\end{array}$ & $\begin{array}{l}\text { Adjusted OR (Cl) } \\
n=435\end{array}$ \\
\hline \multicolumn{7}{|l|}{ Predisposing variables } \\
\hline Age & $0.98(0.95-1.01)$ & & $0.98(0.94-1.02)$ & & $0.97(0.93-1.01)$ & $0.95(0.90-1.00)$ \\
\hline \multicolumn{7}{|l|}{ Level of Education ${ }^{a}$} \\
\hline Low & 1.00 (ref.) & & 1.00 (ref.) & & 1.00 (ref.) & \\
\hline Middle & $1.13(0.70-1.83)$ & & $1.66(0.74-3.71)$ & & $0.91(0.49-1.69)$ & \\
\hline High & $1.06(0.65-1.73)$ & & $1.47(0.65-3.31)$ & & $0.91(0.48-1.71)$ & \\
\hline \multicolumn{7}{|l|}{ Ethnic Background ${ }^{a}$} \\
\hline Dutch & 1.00 (ref.) & & 1.00 (ref.) & & 1.00 (ref.) & \\
\hline Western Non-Dutch & $0.57(0.31-1.04)$ & & $0.74(0.34-1.59)$ & & $0.46(0.16-1.26)$ & \\
\hline Non-Western Non-Dutch & $0.78(0.58-1.01)$ & & $1.03(0.66-1.59)$ & & $0.58(0.38-0.88)$ & \\
\hline \multicolumn{7}{|l|}{ Occupation $^{\mathrm{a}}$} \\
\hline Unemployed & 1.00 (ref.) & & 1.00 (ref.) & & 1.00 (ref.) & \\
\hline Employed & $1.03(0.76-1.41)$ & & $1.07(0.68-1.69)$ & & $0.99(0.64-1.52)$ & \\
\hline \multicolumn{7}{|l|}{ Enabling variables } \\
\hline \multicolumn{7}{|l|}{ Deprived area ${ }^{a}$} \\
\hline No & 1.00 (ref.) & 1.00 (ref.) & 1.00 (ref.) & & 1.00 (ref.) & 1.00 (ref.) \\
\hline Yes & $0.76(0.52-1.10)$ & $0.64(0.42-0.99)$ & $1.01(0.58-1.75)$ & & $0.55(0.33-0.92)$ & $0.50(0.27-0.92)$ \\
\hline \multicolumn{7}{|l|}{ Team } \\
\hline 1 & 1.00 (ref.) & & 1.00 (ref.) & & 1.00 (ref.) & \\
\hline 2 & $0.76(0.57-1.02)$ & & $0.71(0.47-1.09)$ & & $0.79(0.53-1.17)$ & \\
\hline \multicolumn{7}{|l|}{ Year } \\
\hline 2015 & 1.00 (ref.) & 1.00 (ref.) & 1.00 (ref.) & & 1.00 (ref.) & 1.00 (ref.) \\
\hline 2016 & $1.34(1.01-1.79)$ & $1.56(1.12-2.18)$ & $1.07(0.70-1.62)$ & & $1.64(1.09-2.46)$ & $2.42(1.45-4.05)$ \\
\hline \multicolumn{7}{|l|}{ Need variables } \\
\hline \multicolumn{7}{|l|}{ Parity } \\
\hline Nulliparous & 1.00 (ref.) & & 1.00 (ref.) & & 1.00 (ref.) & \\
\hline Multiparous & $0.86(0.73-1.03)$ & & $0.87(0.66-1.14)$ & & $0.83(0.66-1.05)$ & \\
\hline \multicolumn{7}{|l|}{ Start of pregnancy ${ }^{a}$} \\
\hline Spontaneous & 1.00 (ref.) & 1.00 (ref.) & 1.00 (ref.) & & 1.00 (ref.) & \\
\hline Assisted & $1.56(1.14-2.15)$ & $1.49(1.04-2.13)$ & $1.32(0.73-2.37)$ & & $1.60(1.08-2.36)$ & \\
\hline Duration of getting pregnant ${ }^{a}$ & $1.01(1.00-1.02)$ & & $1.01(0.99-1.04)$ & & $1.00(0.98-1.02)$ & \\
\hline \multicolumn{7}{|c|}{ Attendance general practitioner } \\
\hline No & 1.00 (ref.) & & 1.00 (ref.) & & 1.00 (ref.) & \\
\hline Yes & $1.43(0.96-2.12)$ & & $1.17(0.62-2.20)$ & & $1.54(0.92-2.58)$ & \\
\hline \multicolumn{7}{|l|}{ Attendance Specialist } \\
\hline No & 1.00 (ref.) & & 1.00 (ref.) & & 1.00 (ref.) & \\
\hline Yes & $1.35(0.99-1.84)$ & & $1.08(0.67-1.75)$ & & $1.49(0.98-2.26)$ & \\
\hline \multicolumn{7}{|l|}{ Consultation Obstetrician $^{\mathrm{a}}$} \\
\hline No & 1.00 (ref.) & 1.00 (ref.) & 1.00 (ref.) & 1.00 (ref.) & 1.00 (ref.) & 1.00 (ref.) \\
\hline Yes & $3.46(2.50-4.79)$ & $3.39(2.37-4.85)$ & $3.74(2.26-6.18)$ & $3.99(2.35-6.77)$ & $3.52(2.28-5.45)$ & $2.75(1.66-4.57)$ \\
\hline Psychosocial problems $^{\mathrm{a}}$ & 1.00 (ref.) & & 1.00 (ref.) & & 1.00 (ref.) & 1.00 (ref.) \\
\hline No & $1.53(1.07-2.17)$ & & $1.24(0.75-2.07)$ & & $1.85(1.13-3.03)$ & $1.85(1.02-3.35)$ \\
\hline
\end{tabular}


Table 4 Unadjusted and adjusted associations of various characteristics of women with adequate plus use of care, and split by subsequent referral in the study practice (Continued)

\begin{tabular}{|c|c|c|c|c|c|c|}
\hline & \multicolumn{2}{|l|}{ Study population } & \multicolumn{2}{|l|}{ Not referred } & \multicolumn{2}{|l|}{$\underline{\text { Referred }}$} \\
\hline & $\begin{array}{l}\text { Unadjusted OR (CI) } \\
n \leq 1053^{\mathbf{a}}\end{array}$ & $\begin{array}{l}\text { Adjusted OR (Cl) } \\
n=937\end{array}$ & $\begin{array}{l}\text { Unadjusted OR (CI) } \\
n \leq 560^{\mathbf{a}}\end{array}$ & $\begin{array}{l}\text { Adjusted OR (CI) } \\
n=502\end{array}$ & $\begin{array}{l}\text { Unadjusted OR (Cl) } \\
n \leq 493^{\mathbf{a}}\end{array}$ & $\begin{array}{l}\text { Adjusted OR (Cl) } \\
n=435\end{array}$ \\
\hline Yes in the past & $2.69(1.70-4.25)$ & & $1.28(0.56-2.93)$ & & $3.85(2.13-6.93)$ & $2.99(1.43-6.25)$ \\
\hline \multicolumn{7}{|l|}{ Yes at present } \\
\hline \multicolumn{7}{|c|}{ Sexual or domestic violence ${ }^{a}$} \\
\hline No & 1.00 (ref.) & 1.00 (ref.) & 1.00 (ref.) & 1.00 (ref.) & 1.00 (ref.) & \\
\hline Yes & $2.27(1.55-3.33)$ & $2.25(1.44-3.49)$ & $1.92(1.06-3.48)$ & $2.17(1.11-4.24)$ & $2.43(1.46-4.03)$ & \\
\hline \multicolumn{7}{|l|}{ Prescribed Drugs ${ }^{\mathrm{a}}$} \\
\hline No & 1.00 (ref.) & & 1.00 (ref.) & & 1.00 (ref.) & \\
\hline Yes & $1.42(0.89-2.26)$ & & $1.29(0.59-2.81)$ & & $1.36(0.75-2.46)$ & \\
\hline \multicolumn{7}{|c|}{ Number of ultrasounds } \\
\hline (mean) & $1.18(1.07-1.31)$ & $1.18(1.05-1.33)$ & $1.09(0.94-1.27)$ & & $1.27(1.11-1.46)$ & $1.38(1.15-1.65)$ \\
\hline \multicolumn{7}{|c|}{ Health behaviour variables } \\
\hline \multicolumn{7}{|l|}{ Smoking $^{a}$} \\
\hline No & 1.00 (ref.) & 1.00 (ref.) & 1.00 (ref.) & & 1.00 (ref.) & \\
\hline Yes & $1.81(1.32-2.50)$ & $1.59(1.10-2.30)$ & $1.81(1.13-2.90)$ & & $1.75(1.13-2.71)$ & \\
\hline \multicolumn{7}{|l|}{$B M l^{\mathrm{a}}$} \\
\hline Normal weight & 1.00 (ref.) & 1.00 (ref.) & 1.00 (ref.) & & 1.00 (ref.) & 1.00 (ref.) \\
\hline Overweight & $1.34(0.93-1.86)$ & $1.51(1.04-2.18)$ & $1.02(0.61-1.70)$ & & $1.52(0.96-2.39)$ & $1.88(1.09-3.24)$ \\
\hline Obese & $0.96(0.61-1.50)$ & $0.80(0.47-1.35)$ & $0.61(0.25-1.48)$ & & $1.02(0.59-1.77)$ & $0.79(0.39-1.58)$ \\
\hline
\end{tabular}

a sample size varies due to missing data; $O R$ odds ratio, $\mathrm{Cl}$ confidence intervals $95 \%$; education: low (primary school and some vocational training), intermediate (secondary school and completed vocational training), and high (college and/or university)

A majority of frequent attenders had complaints related to psycho-social problems, a broader definition including everything that affects the functioning of woman's daily life such as worries or having vague complaints [21], one of the perceived needs according to Andersen's model; medical reasons were less identified. This observation is in accordance with previous studies in general practice $[8,22]$. Pregnancy-related anxiety, defined as worries and fears related to the health of the child, giving birth, parenting and/or caring for the child, is an important psychological factor [23, 24]. Most pregnant women report pregnancy-related anxiety, which may be in the continuum of normality since it is an indicator of an important motherhood task of protecting and caring for the unborn baby [24]. Nevertheless, higher levels of pregnancy-related anxiety is a known risk factor for adverse perinatal outcomes [25-28]. Preterm birth, low birth weight, perinatal mortality, and lower Apgar scores are also related to frequent attendance [1113].

Even though medical reasons were less reported as an underlying cause, frequent attenders did often have consultations with an obstetrician, one of the evaluated needs according to Andersen's model. These consultations were mainly because of medical reasons such as decrease in fetal growth, less fetal movements, and blood loss. The beforementioned symptoms are related to fetal distress and obstetricians could more carefully monitor the condition of the fetus for the best possible outcomes.

Finally, we found a strong association between frequent attendance and a history of sexual violence, one of the perceived needs according to Andersen's model. Sexual and domestic violence is an important problem worldwide. The prevalence of sexual violence under the age of 18 is over $20 \%$, with even a higher prevalence across all ages [29, 30]. The reported prevalence of sexual violence during pregnancy is $17 \%$ while only a third of the women disclose the sexual violence to their caregiver [31, 32]. Compared to women without a history of sexual violence, women exposed to sexual violence are more likely to fear child birthing, have negative birth experiences and adverse perinatal outcomes [31, 33]. Building trusting relationships with women is of importance to improve care and to facilitate births in a sensitive way so that re-enactment of abuse will be minimized [1]. In 2017, 
the \#metoo movement started a public confession about the sexual violence of famous and non-famous women. As a subsequent result, a public debate on this subject started in many countries. The \#MeToo movement is of great importance to provide a safe and supportive environment where sexual violence could be openly discussed, including in maternity/prenatal care. As only about a third of pregnant women reveal sexual violence to their caregiver, we expect an underreporting of women who experienced sexual violence. Therefore this subject should be discussed on a regular basis throughout the pregnancy. We also recommend being alert to physical complaints such as chronic pelvic pain, as these could indicate a history of sexual violence [34].

To the best of our knowledge, this is the first study to explore the phenomenon of frequent attendance in primary midwifery care. The dataset was robust with detailed and unique information; de-identified and anonymized data were extracted from the digital midwifery database including medical charts, containing information concerning sexual violence and psychosocial problems. These facts are not available in national registrations of birth outcomes. The study cohort was similar to the Dutch national pregnant population women with respect to average age (29.2 years versus 31.1 years), and proportion of primipara (29.2 years versus 31.1 years) [17]. However, the proportion of non-Dutch women in our study was significantly higher $(53 \%$ versus $26 \%$ ); this could explain the higher prevalence of referral compared to the national pregnant population (47 and 35\%) [17]. Furthermore, our sample size was large enough to examine the distinction between referred and non-referred pregnant women and showed that these two groups had different medical and non-medical problems that needed more care provided by the midwives.

The performance statistics of the regression model on the subset of referred participants indicate that this model could explain well the occurrence of frequent attendance and that the results could be generalized to the underlying population of pregnant women in prenatal care. In contrast, the performance statistics of the regression models on the entire study population and the subset of non-referred participants indicated that these models could not completely explain the occurrence of frequent attendance and could not be generalized to the underlying population of pregnant women in prenatal care. Different models might be obtained with a larger and different study population, adjusting for several relevant factors that were not included in this study [35, 36]; e.g. mental health was not included in our study, whereas women with a mental disability have more prenatal visits compared to women without [37].

Our research recommendation is to repeat this study in a larger database that includes multiple practices to make the results more generalizable. In addition, we recommend exploring, through qualitative research, women's underlying reasons and perceptions regarding the use of prenatal health care. This would add information for policymakers in order to adjust prenatal guidelines to the needs of pregnant women.

\section{Conclusions}

In our exploratory study of low-risk women, frequent attendance was observed in almost one in four pregnant women mainly because of non-medical reasons. Associations with frequent attendance differ between non-referred and referred pregnant women. Non-referred frequent attenders had relatively more a history of sexual violence, whereas referred frequent attenders had relatively more often psychosocial problems.

\section{Implications for practice}

To provide the best possible care, a trustful midwife-woman relationship is needed for women to share more details in case of vague complaints or worries to identify their implicit needs. Furthermore, the presence of sexual violence should be explored at least twice during pregnancy, because only a third of women disclose a history of sexual violence during pregnancy. Such information can be used by midwives to attune the prenatal care to the woman's specific needs. This implies that (student-)midwives have to learn to be flexible with the guidelines about the prenatal visits schedule and have to decide together with every individual pregnant woman when and how prenatal care is provided.

\section{Appendix}

Kotelchuck Index-Revised. Index for assessment of the adequacy of prenatal care utilization in the Dutch primary midwifery care context.

This index, based on the Kotelchuck Index, is a validated tool for measuring the level of care utilization, is based on the number of face-to-face visits with the midwife, adjusted for gestation and onset of care and aligned with the number of recommended face-to-face prenatal visits by the KNOV. Utilization of care was defined as inadequate (less than $50 \%$ of the recommended visits), intermediate $(50-79 \%)$, adequate $(80-109 \%)$ and adequate plus (110\% or more). 
Table $\mathbf{5}$ Index for assessment of the adequacy of prenatal care utilization in the Dutch primary midwifery care context [3]

\begin{tabular}{|c|c|c|c|}
\hline $\begin{array}{l}\text { Duration of } \\
\text { gestation }\end{array}$ & $\begin{array}{l}\text { Initiation of } \\
\text { care }\end{array}$ & $\begin{array}{l}\text { Number of } \\
\text { visits }\end{array}$ & $\begin{array}{l}\text { Kotelchuck } \\
\text { Index }^{b}\end{array}$ \\
\hline \multirow[t]{4}{*}{$0-11+6$} & \multirow[t]{4}{*}{$\leq 11+6$} & $\geq 3$ & 4 \\
\hline & & $1-2$ & 3 \\
\hline & & & 2 \\
\hline & & 0 & 1 \\
\hline \multirow{5}{*}{$\begin{array}{l}12-26+6 \\
\text { Ideally } 3.75 \text { visits }^{a}\end{array}$} & \multirow[t]{4}{*}{$\leq 11+6$} & $\geq 6$ & 4 \\
\hline & & $3-5$ & 3 \\
\hline & & 2 & 2 \\
\hline & & $\leq 1$ & 1 \\
\hline & \multirow[t]{5}{*}{$\geq 12+0$} & & 1 \\
\hline \multirow{5}{*}{ 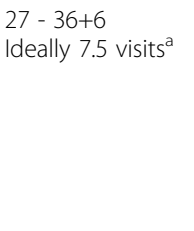 } & & $\geq 10$ & 4 \\
\hline & & $6-9$ & 3 \\
\hline & & $4-5$ & 2 \\
\hline & & $\leq 3$ & 1 \\
\hline & $\geq 12+0$ & & 1 \\
\hline \multirow{5}{*}{ 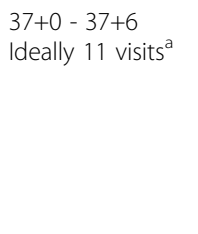 } & \multirow[t]{4}{*}{$\leq 11+6$} & $\geq 13$ & 4 \\
\hline & & $10-12$ & 3 \\
\hline & & $6-9$ & 2 \\
\hline & & $\leq 5$ & 1 \\
\hline & $\geq 12+0$ & & 1 \\
\hline \multirow{5}{*}{$\begin{array}{l}38+0-38+6 \\
\text { Ideally } 12 \text { visits }^{\text {a }}\end{array}$} & \multirow[t]{4}{*}{$\leq 11+6$} & $\geq 14$ & 4 \\
\hline & & $10-13$ & 3 \\
\hline & & $6-9$ & 2 \\
\hline & & $\leq 5$ & 1 \\
\hline & $\geq 12+0$ & & 1 \\
\hline \multirow{5}{*}{$\begin{array}{l}39+0-39+6 \\
\text { Ideally } 13 \text { visits }^{a}\end{array}$} & \multirow[t]{4}{*}{$\leq 11+6$} & $\geq 15$ & 4 \\
\hline & & $11-14$ & 3 \\
\hline & & $7-10$ & 2 \\
\hline & & $\leq 6$ & 1 \\
\hline & $\geq 12+0$ & & 1 \\
\hline \multirow{5}{*}{$\begin{array}{l}40+0-40+6 \\
\text { ldeally } 14 \text { visits }^{a}\end{array}$} & \multirow[t]{4}{*}{$\leq 11+6$} & $\geq 16$ & 4 \\
\hline & & $12-15$ & 3 \\
\hline & & $7-11$ & 2 \\
\hline & & $\leq 6$ & 1 \\
\hline & $\geq 12+0$ & & 1 \\
\hline \multirow{5}{*}{ 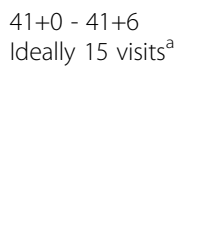 } & \multirow[t]{4}{*}{$\leq 11+6$} & $\geq 17$ & 4 \\
\hline & & $12-16^{*}$ & 3 \\
\hline & & $8-11$ & 2 \\
\hline & & $\leq 7$ & 1 \\
\hline & $\geq 12+0$ & & 1 \\
\hline
\end{tabular}

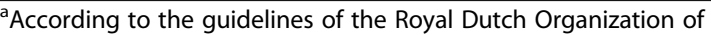
Midwives

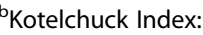

1. Inadequate (received less than $50 \%$ of expected visits)

2. Intermediate (50\%-79\%)

3. Adequate (80\%-109\%)

4. Adequate Plus ( $110 \%$ and more)

\section{Abbreviations}

KNOV: Koninklijke Organisatie van Verloskundigen - the Royal Dutch

Organisation of Midwives; POP: Psychiatrist-Obstetrician-Pediatrician-clinic

\section{Acknowledgments}

Nothing to acknowledge.

\section{Funding}

The study has been funded by the Royal Dutch Organization of Midwives (KNOV) Fellowship 2017. The KNOV had no involvement of the funders in the study design, data analyses, manuscript preparation, and publication decisions.

\section{Availability of data and materials}

The datasets used and/or analysed during the current study are available from the corresponding author on reasonable request.

\section{Authors' contributions}

$J G, B W$ and LM designed the study with the support of EF. JG and BW collected the data. JG and LG, and EF conducted the statistical analyses independently and discussed the slight differences in outcomes. JG, LG en $\mathrm{AH}$ discussed the interpretations. JG drafted the manuscript with the advice and input of $L G, A H, L M$ and $E F$, and all authors read and corrected draft versions of the manuscript and approved the final manuscript.

\section{Ethics approval and consent to participate}

On the 25th of January 2018, the Medical Ethics Committee of the VU University Medical Center (ref. 2018.019) approved this study to collect and analyse retrospective data of January 2015 to January 2017. Women gave permission to use their anonymous data, their verbal consent was obtained during the intake and noted in their files while they watched; the beforementioned Medical Ethics Committee approved this procedure of obtaining consent.

\section{Consent for publication}

Not applicable.

\section{Competing interests}

The work of Dr. Janneke Gitsels-van der Wal was funded by KNOV Fellowship 2017. The work of the other authors was not funded and they have nothing to disclose.

\section{Publisher's Note}

Springer Nature remains neutral with regard to jurisdictional claims in published maps and institutional affiliations.

\section{Author details}

${ }^{1}$ Amsterdam UMC, Vrije Universiteit Amsterdam, Midwifery Science, AVAG, Amsterdam Public Health research institute, De Boelelaan 1117, Amsterdam, The Netherlands. ${ }^{2}$ ESRC funded Business and Local Government Data Research Centre (BLG DRC), School of Computing Sciences, University of East Anglia, Norwich Research Park, Norwich NR4 7TJ, England, UK. ${ }^{3}$ Department of Obstetrics and Gynaecology, Amsterdam UMC, Vrije Universiteit Amsterdam, De Boelelaan 1117, Amsterdam, Netherlands. ${ }^{4}$ Department of Obstetrics and Gynecology, Zaans Medical Center, Koningin Julianaplein 58, Zaandam, Netherlands. ${ }^{5}$ Department of General Practice \& Elderly Care Medicine, University of Groningen, University Medical Center Groningen, PO Box 30001, 9700 RB Groningen, the Netherlands.

Received: 17 December 2018 Accepted: 25 April 2019

Published online: 17 June 2019

\section{References}

1. World Health Organisation - recommendations on antenatal care for a positive pregnancy experience. 2016. Available from: http://apps.who.int/iris/bitstream/ handle/10665/250796/9789241549912-eng.pdf;jsessionid= A589CE249315348D20CBB3E1D536A6AE?sequence=1. Accessed 23 Apr 2019.

2. NICE National Institute for Health and Care Excellence - guideline Antenatal care for uncomplicated pregnancies: schedule of appointments. Available from: https://pathways.nice.org.uk/pathways/antenatal-care-foruncomplicated-pregnancies\#path=view\%3A/pathways/antenatal-care-for- 
uncomplicated-pregnancies/antenatal-care-for-uncomplicated-pregnanciesschedule-of-appointments.xm|\&content=view-index. Accessed 23 Apr 2019.

3. Feijen-de Jong E, Jansen DWMC, Baarveld F, Boerleider A, Spelten E, Schellevis F, Reijneveld SA. Determinants of prenatal healthcare utilization by low-risk women in primary midwifery-led care in the Netherlands: a prospective cohort study. Women Birth. 2015;28(2):87.

4. Ministry of Health. Demographic and Health Survey of Indonesia 2012. Available from: https://dhsprogram.com/pubs/pdf/fr275/fr275.pdf.

5. De Boer, J., Zeeman, K. Prenatale verloskundige begeleiding. Aanbevelingen voor ondersteuning, interactie en voorlichting. KNOV-standaard Wetenschappelijke onderbouwing, 2008 Utrecht. In Dutch. Available from: https://www.knov.nl/serve/file/knov.nl/knov_downloads/985/file/ Wetenschappelijke\%20onderbouwing\%20standaard\%20prenatale \%20verloskundige\%20begeleiding.pdf. Accessed 17 May 2019.

6. Hildingsson I, Waldenström U, Rådestad I. Women's expectations on antenatal care as assessed in early pregnancy: number of visits, continuity of caregiver and general content. Acta Obstet Gyn Scan. 2002;81:118.

7. Hildingsson I, Rådestad I, Waldenström U. Number of antenatal visits and women's opinion. Acta Obstet Gyn Scan. 2005;84:248.

8. Smits FT, Brouwer HJ, Zwinderman AH, Mohrs J, Schene AH, van Weert HC, ter Riet G. Why do they keep coming back? Psychosocial etiology of persistence of frequent attendance in primary care: A prospective cohort study. J Psychosom Res. 2014;77(6):492.

9. Hajek A, Bock J-O, König H-H. Association of general psychological factors with frequent attendance in primary care: a population-based crosssectional observational study. BMC Fam Pract. 2017;18:48.

10. Smits FTM, Mohrs JJ, Beem EE, Bindels PJE, van Weert HCPM. Defining frequent attendance in general practice. BMC Fam Pract. 2008;9:21.

11. Hemminki E, Gissler M. Quantity and targeting of antenatal care in Finland. Acta Obstet Gyn Scan. 1993;72:24.

12. Kotelchuck M. An evaluation of the Kessner Adequacy of Prenatal Care Index and proposed Adequacy of Prenatal Care Utilization Index. Am J Public Health. 1994;84:1414.

13. De Jonge A, Rijnders M, Agyemang C, van der Stouwe R, den Otter J, Van den Muijsenbergh ME, Buitendijk S. Limited midwifery care for undocumented women in the Netherlands. J Psychosom Obst Gyn. 2011;32(4):182.

14. Carter EB, Tuuli MG, Caughey AB, Odibo AO, Macones GA, Cahill AG. Number of prenatal visits and pregnancy outcomes in low-risk women. J Perinat. 2016;36(3):178.

15. Andersen RM, Rice TH, Kominkski GF. Changing the U.S. health care system; key issues in health services policy and management. San Francisco: Jossey-Bass; 2007.

16. World Health Organisation - BMl. Available from: http:/www.euro.who.int/ en/health-topics/disease-prevention/nutrition/a-healthy-lifestyle/body-massindex-bmi. Accessed 16 Dec 2018

17. Perined. Perinatal Care in the Netherlands 2016. In Dutch, available from: https://assets.perined.nl/docs/7935f9c6-eaac-4f59-a150-307ae04efa27.pdf. Assessed 16 Dec 2018

18. Peristat II: EURO-PERISTAT project in collaboration with SCPE, EUROCAT and EURONEONET. European perinatal health report. Better statistics for better health for pregnant women and their babies in 2004. 2008. Available from: www.europeristat.com. Accessed 16 Dec 2018.

19. Report A good start (Een goed begin) in Dutch. 2009. Available from: https://www.nvog.nl/wp-content/uploads/2018/02/Advies-Stuurgroepzwangerschap-en-geboorte-1.0-01-01-2009.pdf. Accessed 17 May 2019.

20. Choté AA, de Boer C, Bruijnzeels MA, Redekop K, Jaddoe WW, Hofman A, Steegers EA, Mackenbach JP, Foets M. Ethnic differences in antenatal care use in a large multi-ethnic urban population in the Netherlands. Midwifery. 2011;27(1):36.

21. http://www.euprimarycare.org/sites/default/files/

Prevalence\%20and\%20management\%20of\%20psychosocia \%20problems\%20in\%20primary.pptx (broader definition of psychosocial problems). Accessed 17 May 2019.

22. Smits FT, Brouwer HJ, ter Riet G, van Weert HCP. Epidemiology of frequent attenders: A 3-year historic cohort study comparing attendance, morbidity and prescriptions of one-year and persistent frequent attenders. BMC Public Health. 2009;9:36.

23. Bayrampour H, Ali E, McNeil DA, Benzies K, MacQueen G, Tough S. Pregnancy-related anxiety: A concept analysis. International Journal of Nursing Studies. 2016;55:115-30.

24. Harpel TS. Fear of the unknown: Ultrasound and anxiety about fetal health. Health (London). 2008;12(3):295.
25. Dunkel Schetter C, Tanner L. Anxiety, depression and stress in pregnancy: Implications for mothers, children, research, and practice. Curr Opin Psychiatr. 2012;25(2):141.

26. Shapiro GD, Fraser WD, Frasch MG, Séguin JR. Psychosocial stress in pregnancy and preterm birth: Associations and mechanisms. J Perinat Med. 2013;41(6):631.

27. Loomans EM, Van Dijk AE, Vrijkotte TG, van Eijsden M, Stronks K, Gemke RJ, van den Bergh BR. Psychosocial stress during pregnancy is related to adverse birth outcomes: Results from a large multi-ethnic community-based birth cohort. Eur J Public Health. 2013;23(3):485.

28. Gonzalez-Mesa E, Arroyo-Gonzalez M, Ibrahim-Diez N, Cazorla-Granados O. Mood state at the beginning of the pregnancy and its influence on obstetric and perinatal outcomes. J Psychosom Obst Gynecol. 2018;1:1.

29. Pereda N, Guilera G, Forns M, Gomez-Benito J. The prevalence of child sexual abuse in community and student samples: a meta-analysis. Clin Psychol Rev. 2009;29(4):328.

30. Okur P, van der Knaap L, Bogaerts S. Prevalence and nature of child sexual abuse in the Netherlands: ethnic differences? J Child Sex Abus. 2015;24(1):1.

31. Montgomery E, Pope $C$, Rogers J. The re-enactment of childhood sexual abuse in maternity care: a qualitative study. BMC Pregnancy Childb. 2015;26(15):194.

32. Bazyar J, Safarpour H, Daliri S, Karimi A, Safi Keykaleh M, Bazyar M. The prevalence of sexual violence during pregnancy in Iran and the world: a systematic review and meta-analysis. Violence Res. 2018;10(2):63-73.

33. Leeners B, Stiller R, Block E, Hemgartner MP. Pregnancy complications in women with childhood sexual abuse experiences. Journal of Psychosom Res. 2010;69(5):503.

34. Hooker $A B$, van Moorst $B R$, van Haarst EP, van Ootegehem NA, van Dijken DK, Heres MH. Chronic pelvic pain: evaluation of the epidemiology, baseline demographics, and clinical variables via a prospective and multidisciplinary approach. Clin Exp Obstet Gyn. 2013;40(4):492.

35. Westert GP, Groenewoud S, Wennberg JE, Gerard C, Dasilva P, Atsma F, Goodman DC. Medical practice variation: public reporting a first necessary step to spark change. Int J Qual Health C. 2018;1;30(9):731-735.

36. Seijmonsbergen-Schermers AE, Zondag DC, Nieuwenhuijze $M$, van den Akker T, Verhoeven CJ, Geerts C, Schellevis F, de Jonge A. Regional variations in childbirth interventions in the Netherlands: a nationwide explorative study. BMC Pregnancy Childb. 2018;18:192.

37. Redshaw M, Malouf R, Gao H, Gray R. Women with disability: the experience of maternity care during pregnancy, labour and birth and the postnatal period. BMC Pregnancy Childb. 2013;13:174.

\section{Ready to submit your research? Choose BMC and benefit from:}

- fast, convenient online submission

- thorough peer review by experienced researchers in your field

- rapid publication on acceptance

- support for research data, including large and complex data types

- gold Open Access which fosters wider collaboration and increased citations

- maximum visibility for your research: over $100 \mathrm{M}$ website views per year

At $\mathrm{BMC}$, research is always in progress.

Learn more biomedcentral.com/submissions 